\title{
Pain and Ethnicity in Athletes
}

By: Barton P. Buxton, David H. Perrin, Ronald K. Hetzler, Kwok W. Ho, and Joe H. Gieck*

Buxton, B.P., Perrin, D.H., Hetzler, R.K., Ho, K.W., \& Gieck, J.H. (1993). Pain and ethnicity in athletes. Journal of Sport Rehabilitation, 2, 13-19.

\section{***Note: Figures may be missing for this format of the document ***Note: Footnotes and endnotes indicated with parentheses}

\begin{abstract}
:
The purpose of this investigation was to determine the relationship between ethnicity and acute pain response in male athletes. Subjects included 93 male athletes (age $=18.65 \pm .58$ years) of differing ethnicity. Each subject performed a Cold Pressor Test (CPT) and was evaluated for pain threshold and pain tolerance times. Two one-way analyses of variance were performed to analyze the data. The results indicated that significant differences existed in pain tolerance times between ethnic groups ( $\mathrm{p}<.05)$. However, no differences were observed in pain threshold times. These findings support the existence of a difference in pain tolerance between ethnic groups in collegiate athletes.
\end{abstract}

\section{Article:}

Although pain is a physiological response, an individual's pain perception has been related to psychological, social, cultural, and ethnic factors (9). The sensation of pain and its secondary dysfunction are primary reasons athletes seek treatment from sports medicine practitioners. To date, little information about the relationship between pain response in collegiate athletes is available in the literature. This is noteworthy because athletes represent a unique group of individuals from varied cultural and ethnic backgrounds who suffer from a variety of injuries due to training and competition. Because the majority of these athletes are young and otherwise healthy, they may respond to acute pain differently than do the varied cultural and ethnic populations previously studied.

The relationship between ethnicity and pain expression has interested investigators since the early 1950s. Zborowski (13) compared the differences in pain expression among four ethnic groups: Irish, Italian, Jewish, and Old American (native Anglo-Saxon) in a New York City hospital. The expression of pain was measured empirically on the basis of interviews with the patients. The reported findings indicated that Jewish and Italian patients were less inhibited in the expression of pain than Irish or Old American. In 1966, Zola (14) compared ethnic background and response to pain for subjects of Italian Catholic, Irish Catholic, and AngloSaxon backgrounds; this researcher also measured the response to pain by interviewing the patients. The reported findings indicated that there was a latency for pain expression among the

\footnotetext{
* Buxton is with the University of Hawaii at Manoa, Department of Health, Physical Education and Recreation, College of Education, and The John A. Burns School of Medicine, 1337 Lower Campus Road, Honolulu, HI 96822. Hetzler and Ho are with the University of Hawaii at Manoa, Department of Health, Physical Education and Recreation, College of Education, 1337 Lower Campus Road, Honolulu, HI 96822. Perrin and Gieck are with the University of Virginia, Department of Health, Physical Education and Recreation, Memorial Gymnasium, Charlottesville, VA 22903.
} 
Irish and Anglo-Saxon patients but that the expression of pain was less inhibited among the Italian patients. In contrast, Flannery et al. (3) indicated no significant differences in pain expression between Black, Irish, Italian, Jewish, and Protestant Anglo-Saxon women following childbirth (with episiotomy). Flannery et al. evaluated the personal perceptions of pain by administering a self-assessment tool and by behavioral observations. More recently, Greenwald (5) reported ethnic differences in pain perception using a graphic rating scale and the McGill Pain Questionnaire (8) in 536 subjects with various forms of cancer. The findings of this recent investigation support the previous observations $(2,13,14)$ that cultures may be associated with specific ethnicity in the expression of pain. In addition, other studies have reported differences in pain expression among ethnic racial populations under varied conditions $(4,6,11)$. The assumption that ethnicity and culture affect an individual's response to pain has been demonstrated with a variety of methods $(4,5,11,13,14)$. However, to our knowledge, no one has investigated the ethnic differences in the expression of pain in collegiate athletes. Therefore, the purpose of this investigation was to determine the relationship between ethnicity and acute pain response in male athletes.

\section{METHODOLOGY}

\section{Subjects}

Subjects included 93 male athletes (age $=18.65 \pm .58$ ). The subjects all participated on intercollegiate athletic teams and were designated into groups by ethnicity. The ethnicity designations included Caucasion $(\mathrm{n}=54)$, Afro-American $(\mathrm{n}=27)$, and Other $(\mathrm{n}=12)$ (Hispanic-Americans and Asian-Americans). The designation of subjects to the Other ethnic group was due to the small number of subjects who represented Hispanic-Americans and AsianAmericans. The subject population was represented by 28 different religious denominations, and over $20 \%$ of the population came from designated minority groups (10).

Only subjects free from history of cardiac and respiratory problems, Raynaud' s disease, frostbite, hypertension, vascular compromise of either upper extremity, injury to either hand, or mental dysfunction were allowed to participate. All subjects read and signed a consent-toparticipate form approved by a university Human Investigation Committee.

\section{The Cold Pressor Test (CPT)}

Individual interpretation of pain will always include some degree of subjectivity. Nevertheless, in an experimental model, researchers need to quantify individual pain responses in a controlled environment to decrease the subjective interpretation of pain expression. The CPT enables the researcher to quantify pain response in terms of pain threshold and pain tolerance time. Therefore, the CPT (7) was used in this investigation as administered and described by Wolff (12). The time elapsed (seconds) from immersion of the hand in ice water to start of the verbal statement "pain" was the pain threshold time. The time elapsed from immersion of the hand in ice water to the start of the verbal statement "stop" was the pain tolerance time. A digital stopwatch with lap counter was used for the timing. The same examiner performed all time testing.

\section{CPT Pretest}


A CPT pretest was administered to each subject $24 \mathrm{hr}$ before the administration of the actual CPT. This procedure allowed the subject to cognitively measure the amount of pain felt in the cold water and become familiar with the experience of cold water pain. The pretest lasted long enough for the subjects to reach their pain tolerance or until $3 \mathrm{~min}$ had elapsed. The pretest was performed on each subject's dominant hand. To further allow the subject to cognitively qualify individual interpretations of the CPT, the pretest included two subjective pain scales (8): a visual analog scale and a pain rating index. The subjects marked a visual analog scale every $15 \mathrm{~s}$ (with a pencil held in the nondominant hand) while the dominant hand was submerged wrist deep in water at $0{ }^{\circ} \mathrm{C}$. When the pretest was concluded, the subjects were asked to place a check mark next to the word or words, on the pain rating index, that best described the pain they were experiencing during the pretest trial. The subjects were told that they were performing a task to identify subjective responses to cold water and not a pretest of the study.

\section{Experimental Trial}

The CPT was performed with each subject alone in a cubical. The sides of the cubical were unmarked. The examiner stood behind and above the cubical during the CPT, so that the test was visually monitored. The subject was unable to see the examiner during the CPT. The subject was seated in a chair with a table placed in front of him. Two plastic 3-gal Igloo® coolers were on the table. The cooler marked I contained warm water maintained at body temperature $\left(37^{\circ} \mathrm{C}\right)$. The cooler marked II contained cold water, saturated with ice shavings, maintained at $0{ }^{\circ} \mathrm{C}$. Water temperature was confirmed with a Celsius thermometer before each CPT measurement.

All instructions were played on a tape recorder to each subject before administration of the CPT to provide standardization. Each subject was then questioned to ensure that he understood. The instructions were subsequently repeated immediately before each CPT measurement. The tape recording requested each subject to place the nondominant hand, wrist deep, into the cooler marked I. The subject was then told to verbally report when the hand was in the warm water. The subject was instructed to keep the hand in the warm water until told to remove it. The period of time in the warm water was $2 \mathrm{~min}$, which allowed for standardization of skin temperature.

When 2 min had expired, each subject was required to place the nondominant hand in the cooler marked II. The subject was told to verbally report when the nondominant hand was immersed up to the wrist by saying "in." The subject was instructed to silently observe the point when the sensation in the hand first became uncomfortable. When the sensation became "definitely painful" (i.e., "it hurts"), the subject was requested to respond with the verbal response "pain." This indicated pain threshold time. Each subject was asked to remain silent and to keep the hand immersed in the water for as long as possible (i.e., until the sensation was unbearable). Each subject was instructed to withdraw the hand from the water when the limit of pain was reached and make the verbal statement "stop." This indicated pain tolerance time.

\section{Reliability of Measures and Statistical Analysis}

Test - retest reliability of the CPT as administered in this study has been reported in the literature (1). Sixteen randomly selected male subjects (mean age 19.75 years) completed a CPT and were subsequently retested 14 days after the original CPT was given. Intraclass correlation coefficients (ICC) reported R values of 0.93 for pain threshold and 0.97 for pain tolerance. 
The statistical analyses in this investigation consisted of computation of two one-way analyses of variance (ANOVA), one between pain threshold and ethnicity and one between pain tolerance and ethnicity. In consideration of disproportional group sizes, a general linear model (GLM) procedure for unbalanced ANOVA was executed with the SAS System. This procedure utilizes Type III sums of squares, which tests the same hypothesis that would have been tested if the data were balanced. The post hoc analysis for multiple comparisons was performed with the Scheffe post hoc test. The alpha level was set at 0.05 .

\section{RESULTS}

The ANOVA indicated significant differences for pain tolerance between ethnic groups ( $\mathrm{p}<.05)$. However, no significant differences were observed for pain threshold between ethnic groups. The statistical results for pain threshold and pain tolerance are presented in Tables 1 and 2, respectively.

Significant differences were observed between the Other group and the Afro-American and Caucasian groups for pain tolerance, with the Scheffe $F$ test used for post hoc multiple comparison $(\mathrm{p}<.05)$. No significant differences were observed between the Afro-American and Caucasian groups (Figure 1). The group containing Asian-American and Hispanic-American subjects (Other ethnic group) had the highest mean $( \pm$ SD) for pain tolerance times (124.95 $\pm 109.21 \mathrm{~s})$. The Caucasian group had the second highest mean $( \pm \mathrm{SD})$ for pain tolerance times (78.33 $\pm 56.89 \mathrm{~s})$, and the Afro-American group had the lowest reported mean $( \pm \mathrm{SD})$ for pain tolerance times $(44.21 \pm 15.81 \mathrm{~s})$. Conversely, the mean $( \pm \mathrm{SD})$ for pain threshold times for the Caucasian group was $22.81( \pm 14.52) \mathrm{s}$, for the Other ethnic group $21.75( \pm 11.52) \mathrm{s}$, and for the Afro-American group $20.08( \pm 10.97) \mathrm{s}$, and no significant differences were observed.

\section{DISCUSSION}

The main finding of the present study was that differences were demonstrated between ethnic groups for acute pain response in male athletes. Specifically, differences were observed between pain tolerance and ethnic groups. Therefore, data from this investigation support the observations of earlier studies in nonathletic populations that reported differences between Afro-American subjects, Caucasians, and varying other ethnic groups with respect to pain expression $(2,3,4,6$, 11). However, the confirmation of these observations with the findings of the earlier studies on pain expression and ethnicity $(4,5,6,11)$ may be limited by the terminology, subject population, and subjective measures of pain.

Table 1

One-Way Analysis of Variance for Pain Threshold and Ethnicity

\begin{tabular}{lrrrr}
\hline Source & \multicolumn{1}{c}{$S S$} & \multicolumn{1}{c}{$d f$} & \multicolumn{1}{c}{$M S$} & $F$ \\
\hline Between groups & 134.46 & 2 & 67.23 & $0.38^{\star}$ \\
Within groups & 15758.97 & 90 & 175.10 & \\
Total & 15893.43 & 92 & & \\
\hline
\end{tabular}

${ }^{*} p=0.68$. 
Table 2

One-Way Analysis of Variance for Pain Tolerance and Ethnicity

\begin{tabular}{lrrrr}
\hline Source & \multicolumn{1}{c}{ SS } & df & \multicolumn{1}{c}{ MS } & $F$ \\
\hline Between groups & 56105.51 & 2 & 28052.75 & $8.17^{*}$ \\
Within groups & 309230.17 & 90 & 3435.89 & \\
Total & 365335.68 & 92 & & \\
\hline
\end{tabular}

$" p=0.0006$

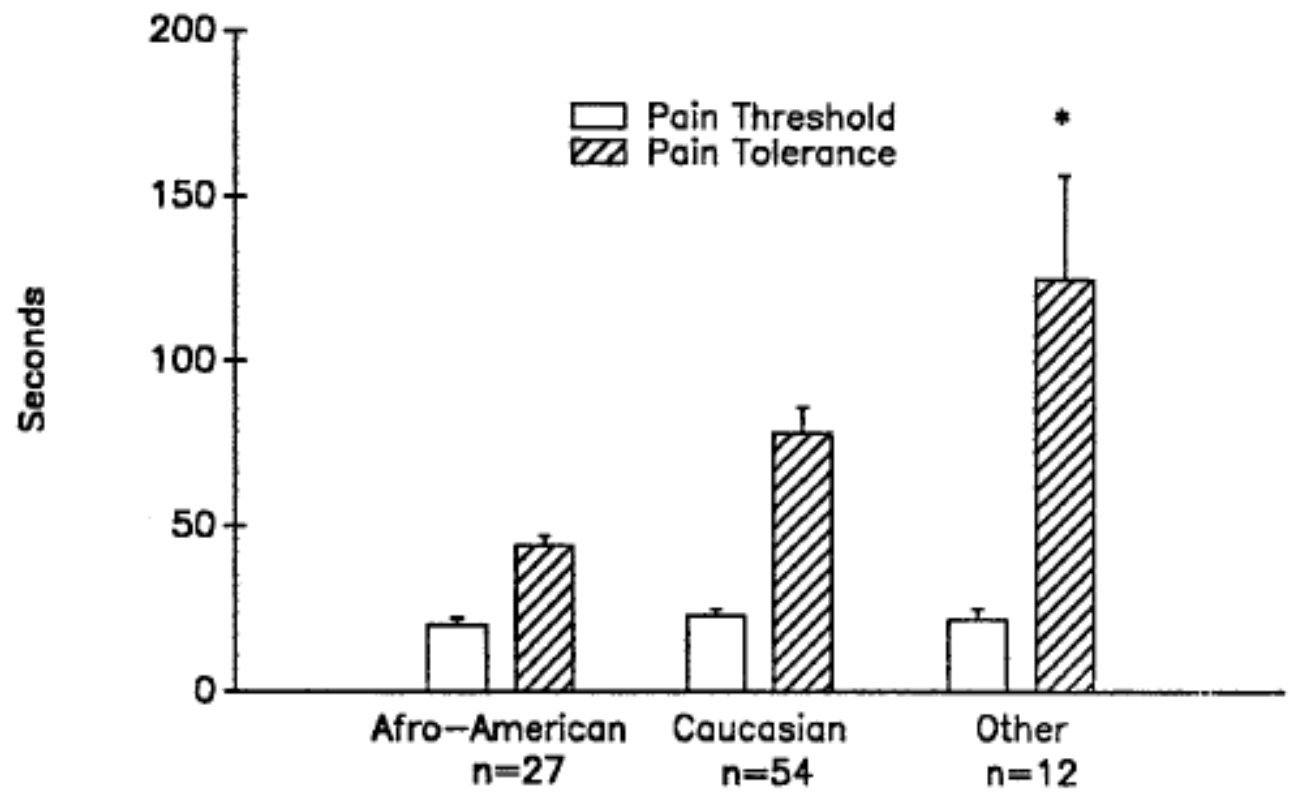

Figure 1: - Pain threshold and pain tolerance times $(\mathrm{M} \pm \mathrm{SE})$ for the three ethnic groups. *p $=$ 0.0006 (Scheffe F test) Other vs. Caucasian and Other vs. Afro-American.

The theory that pain response is related to an individual's sociocultural background has been noted in clinical practice. The assumption that this theory relates to ethnicity is a problematic concern of many health care professionals. The term ethnicity, as used in this study, represents specific racial distinctions between groups. The underlying assumption is that cultural differences in the designated ethnic groups are consistent, although they may be "diluted" or "crosscut" by other types of ethnic background (Italian, Irish, Anglo-Saxon, etc.), as suggested by the work of Greenwald (5).

The issues not withstanding, this investigation found differences between ethnic groups for pain tolerance. However, no significant differences were observed between ethnic groups for pain threshold. This suggests the possibility that there is an ethnic or a cultural variable in the 
individual expression of pain. It is possible that all individuals undergo similar initial responses (threshold) to pain (physiological threat to stasis), regardless of ethnicity or cultural deviation. However, as the pain reaches its extreme limits (tolerance) the ethnic and cultural factors begin to interact, increasing or decreasing the effect related to the perception of pain and its subsequent expression.

What made the present study unique was the utilization of the CPT, which allows the researcher to quantify the acute pain response objectively, in an attempt to control subjectivity in the expression of pain. The CPT was used to induce pain and to measure pain threshold and pain tolerance responses. Moreover, the CPT is easy to administer and its application has limited risks. This is of paramount concern when dealing with human subjects. An additional advantage of the CPT is that it has a low anticipatory effect. The submersion of a subject's nondominant hand (wrist deep) in cold water does not elicit as high an anticipatory effect as does an electrical shock, a spiked pressor gauge, or radiant heat (which can blister skin). Further, the pain threshold and tolerance responses are not affected by skin pigmentation, as they are with radiant heat (2). This was of concern in getting an objective reading on pain threshold and pain tolerance.

A final advantage of the CPT was that the subjects had a high degree of control over the test protocol. Although the subjects were instructed to keep their nondominant hands in the cold water until the sensation became unbearable, the subjects were allowed to remove their hands from the water at any time. This allowed a cognitive judgment about when the pain was at a maximum limit for the subjects. Thus, the subjects were exposed to the stimulus until they could no longer tolerate the sensation and at that time they removed their hands. This differs from analogous forms of pain measurement where subjects are asked to subjectively mark a line or give a number indicating where their pain falls or how much pain they are experiencing. Therefore, the CPT allowed for a quantitative assessment of pain by each subject. Thus, the reported pain threshold and pain tolerance times were objective measures of each subject's pain threshold and pain tolerance.

In conclusion, the purpose of this investigation was to determine the relationship between ethnicity and acute pain response in male athletes. Data from this study support the existence of a relationship between ethnicity and pain tolerance, but not pain threshold, in collegiate male athletes. The complex issue of pain and its secondary dysfunction will continue to challenge the sports medicine practitioner. However, it appears that when treating an athlete in pain the practitioner should be aware that variations in pain expression may exist among ethnic groups.

\section{REFERENCES}

1. Buxton, B.P., and D.H. Perrin. The relationship between personality characteristics and acute pain response in postadolescent males. J. Sport Rehab. 1:111-120, 1992.

2. Chapman, W.P., and C.M. Jones. Variations in cutaneous and visceral pain sensitivity in normal subjects. J. Clin. Invest. 23:81-91, 1944.

3. Flannery, R., et al. Ethnicity as a factor in the expression of pain. Psychosomatics 22:39-50, 1981. 
4. Garron, D., and F. Leavitt. Demographic and affective covariates of pain. Psychosom. Med. 41:525-534, 1979.

5. Greenwald, H.P. Interethnic differences in pain perceptions. Pain 44:157-163, 1991.

6. Greenwald, H.P., J.J. Bonica, and M. Bergner. The prevalence of pain in four cancers. Cancer 60:2563-2569, 1987.

7. Hines, E.A., \& G.E. Brown. Technic of the Cold Pressor Test. Proceed. Staff Meeting Mayo Clin. 14:23, 85-87, 1939.

8. Melzack, R. The McGill Pain Questionnaire: Major properties and scoring methods. Pain $1: 277,1975$.

9. Monks, R., and P. Taenzer. A comprehensive pain questionnaire. In: Pain Measurement and Assessment, R. Melzack (Ed.). New York: Raven Press, 1983, pp. 233-237.

10. Muirhead, G., and D.L. Snyder. Peterson's Guide to Independent Secondary Schools (10th ed). Princeton, NJ: Peterson's Guide Inc., 1988.

11. Wiesburg, M., et al. Pain: Anxiety and Attitudes in Black, White, and Puerto Rican patients. Psychosom. Med. 37:123-135, 1975.

12. Wolff, B.B. Behavioral measurement of human pain. In The Psychology of Pain, R.A. 13. Sternbach (Ed.). New York: Raven Press, 1986, pp. 121-151.

14. Zborowski, M. Cultural components in response to pain. J. Soc. Issues. 8:15-35, 1952.

15. Zola, I. Culture and symptoms - an analysis of patients presenting complaints. Am. Soc. Rev. 31:615-630, 1966. 